Published in final edited form as:

World J Surg. 2015 April ; 39(4): 926-933. doi:10.1007/s00268-014-2899-y.

\title{
Can Focused Trauma Education Initiatives Reduce Mortality or Improve Resource Utilization in a Low-Resource Setting?
}

\section{Robin T. Petroze,}

Department of Surgery, University of Virginia Health System, Charlottesville, VA 22908, USA, rtp3z@virgina.edu

\section{Jean Claude Byiringiro,}

Department of Accident and Emergency, Kigali University Teaching Hospital, Butare, Rwanda

School of Medicine, University of Rwanda, Butare, Rwanda jcbyiringiro@yahoo.fr

\section{Georges Ntakiyiruta,}

Department of Surgery, College of Medicine and Health Sciences, University of Rwanda, Butare, Rwanda, georgentakiyiruta@yahoo.co.uk

\section{Susan M. Briggs,}

Department of Surgery, Massachusetts General Hospital, Boston, MA, USA

Briggs.Susan@mgh.harvard.edu

\section{Dan L. Deckelbaum,}

Centre for Global Surgery, McGill University Health Centre, Montreal, Canada

dan.deckelbaum@muhc.mcgill.ca

\section{Tarek Razek,}

Centre for Global Surgery, McGill University Health Centre, Montreal, Canada

tarek.razek@muhc.mcgill.ca

\section{Robert Riviello,}

Department of Surgery, Brigham and Women's Hospital, Boston, MA, USA

robertriviello@gmail.com

\section{Patrick Kyamanywa,}

Department of Surgery, College of Medicine and Health Sciences, University of Rwanda, Butare, Rwanda pkyamanywa@nur.ac.rw

Jennifer Reid,

School of Medicine, University of Virginia, Charlottesville VA, USA jer4zf@virginia.edu

Robert G. Sawyer, and

Department of Surgery, University of Virginia Health System, Charlottesville, VA 22908, USA, rws2k@virginia.edu

\section{J. Forrest Calland}

\footnotetext{
Correspondence to: J. Forrest Calland, callandevirginia.edu.
}

Conflict of interest None. 
Department of Surgery, University of Virginia Health System, Charlottesville, VA 22908, USA

J. Forrest Calland: calland@virginia.edu

\section{Abstract}

Background-Over $90 \%$ of injury deaths occur in low-income countries. Evaluating the impact of focused trauma courses in these settings is challenging. We hypothesized that implementation of a focused trauma education initiative in a low-income country would result in measurable differences in injury-related outcomes and resource utilization.

Methods-Two 3-day trauma education courses were conducted in the Rwandan capital over a one-month period (October-November, 2011). An ATLS provider demonstration course was delivered to 24 faculty surgeons and 15 Rwandan trauma nurse auditors, and a Canadian Network for International Surgery Trauma Team Training (TTT) course was delivered to 25 faculty, residents, and nurses. Trauma registry data over the 6 months prior to the courses were compared to the 6 months afterward with emergency department (ED) mortality as the primary endpoint. Secondary endpoints included radiology utilization and early procedural interventions. Univariate analyses were conducted using $x^{2}$ and Fisher's exact test.

Results-A total of 798 and 575 patients were prospectively studied during the pre-intervention and post-intervention periods, respectively. Overall mortality of injured patients decreased after education implementation from 8.8 to $6.3 \%$, but was not statistically significant $(p=0.09)$.

Patients with an initial Glasgow Coma Score (GCS) of 3-8 had the highest injury-related mortality, which significantly decreased from $58.5 \%(n=55)$ to $37.1 \%(n=23),(p=0.009$, OR $0.42,95 \% \mathrm{CI} 0.22-0.81$ ). There was no statistical difference in the rates of early intubation, cervical collar use, imaging studies, or transfusion in the overall cohort or the head injury subset. When further stratified by GCS, patients with an initial GCS of 3-5 in the post-intervention period had higher utilization of head CT scans and chest X-rays.

Conclusions-The mortality of severely injured patients decreased after initiation of focused trauma education courses, but no significant increase in resource utilization was observed. The explanation may be complex and multifactorial. Long-term multidisciplinary efforts that pair training with changes in resources and mentorship may be needed to produce broad and lasting changes in the overall care system.

\section{Background}

While trauma exists within every society and socioeconomic strata, death and significant disability due to injuries disproportionately affect the poorer countries of the world where the availability of prevention programs, emergency services, prompt resuscitation, and surgical management are more limited. Over $90 \%$ of injury deaths occur in lowincome countries, and road traffic crashes are one of the most rapidly rising causes of death and disability in low and middle-income countries (LMIC) [1-4].

Addressing early care of the injured patient is fundamental to reducing the global morbidity and mortality due to injuries [5]. In many LMIC, physician shortages as well as material and infrastructure resource limitations challenge the ability to adequately manage trauma in the immediate post-injury period. A variety of educational short-course curricula have been 
developed to teach a systematic approach to the initial management of injured patients in both the pre-hospital and early hospital-based setting [6-9]. Developed in the 1970s, the American College of Surgeons Committee on Trauma's (ACSCOT) Advanced Trauma Life Support (ATLS®) course is the most widely recognized and has been formally used in over 60 countries [10]. Other courses such as the Canadian Network for International Surgery's (CNIS) Trauma Team Training (TTT) course focus on a team-based approach [6]. Limited data exist, however, on the impact of these focused trauma education short-courses on patient outcomes $[11,12]$.

Few LMIC have sustainable or standardized systems available to track patient outcomes. Hospital-based trauma registries are one source of data. Several resource-limited settings have developed and adapted registries for injury surveillance, basic epidemiology, and mortality assessment [13-17]. Modeled after regional registries, a collaborative injury registry was established at the two university referral centers in Rwanda through the University of Rwanda (UR) to collect data on the injured patient population. The registry currently houses more than 8,000 records and accumulates more than 150 new entries per week. This study utilizes prospectively collected data from the Rwanda Injury Registry to study the effects of planned trauma and resuscitation education on patient outcomes and resource utilization.

\section{Methods}

\section{Setting}

Rwanda is a densely populated country in sub-Saharan Africa that has made significant progress in the health and economic sectors since the genocide of 1994. Yet, the population of 11 million is still quite poor with only 5.5 doctors per 100,000 persons [18]. Rwanda is classified by the World Bank as a low-income country; for example, the 2011 gross national income (GNI) per capita was \$570 USD in Rwanda compared to \$48,450 USD in the United States [19].

The Centre Hospitalier Universitaire Kigali (CHUK) is a 520-bed hospital in the capital of Kigali. Since few emergency or surgical services are provided at the district hospitals, injured patients are often sent to the university hospitals for evaluation and definitive care. The hospital is public and serves as one of the main training sites for UR health professional students, including a postgraduate training program in Surgery.

\section{Data collection}

A 31-item, 2-page registry form was locally adapted from registries in Uganda and Tanzania for use in Rwanda [13,14], with data collection beginning in March 2011 at CHUK. Any injured patient who is transferred from a district hospital for evaluation of their injury, any injured patient who dies in emergency from their injury, or any injured patient who is admitted (defined as inpatient hospitalization or emergency stay of $>24 \mathrm{~h}$ ) is prospectively recorded in the registry. Patients who are not transferred from another hospital and are treated and sent home within $24 \mathrm{~h}$ are excluded. Trained nurse registrars in the emergency department (ED) systematically record patient demographics, pre-hospital care, initial 
physiology, early interventions, and patient disposition. Inpatient two-week and 30-day follow-up outcomes are abstracted from patient charts, ward reports, and operating room logs. Data are entered into a computerized, searchable Microsoft Access 2010 (Microsoft Corporation, Santa Rosa, CA) database. Any data extracted for research purposes are deidentified.

\section{Intervention}

Two 3-day trauma education courses were conducted in Kigali over a one-month period (October-November, 2011). An ATLS® provider demonstration course was delivered to 24 faculty surgeons and 15 Rwandan trauma nurse auditors, and a Canadian Network for International Surgery Trauma Team Training (TTT) course was delivered to 25 faculty, residents and nurses.

\section{Data analysis}

Data were exported from the Microsoft Access database and analyzed using SAS 9.3 (SAS Institute, Inc, Cary, NC). As the training sessions took place in Kigali, registry data from CHUK alone was utilized for the analysis. Validated trauma scoring systems such as the Revised Trauma Score and the Kampala Trauma Score [20] were calculated from clinical data collected at admission and used to control for injury severity. Trauma registry data over the 6 months prior to the focused trauma education courses were compared to the 6 months afterward with ED mortality as the primary endpoint. Secondary endpoints included radiology utilization and early procedural interventions. Univariate analyses using $x^{2}$ and Fisher's exact test were conducted to discern statistical significance. A subset cohort analysis was conducted in patients with the highest baseline mortality, those with an initial Glasgow Coma Score (GCS) 3-8. We further stratified all of the results by initial GCS in order to potentially identify the patient subset that could derive the most interventional benefit: GCS 3-5 (severe); GCS 6-8 (severe); GCS 9-12 (moderate); and GCS 13-15 (mild). These strata were based upon categories established in other trauma outcome studies in resource-limited settings [21].

\section{Results}

Phase 1 was the pre-intervention phase (April 9, 2011-October 8, 2011) and had 798 patients with 94 patients, approximately $12 \%$ of the total cohort, included in the subset analysis. Phase 2 was the post-intervention phase (November 21, 2011-May 20, 2012) and had 575 patients with 62 patients, approximately $11 \%$ of the total cohort, included in the subset analysis. There were no significant baseline differences in mechanism of injury, patient demographics, physiology, injury type, or injury severity between the pre- and postintervention groups. Figure 1 details the causes of injury in the two phases. Road traffic crashes caused over $50 \%$ of the injuries in both phases. Falls and blunt force injuries were the next most common, with less than $10 \%$ due to other causes.

Table 1 compares patient characteristics, including physiologic parameters, in the two groups. No significant differences were found in mean pulse, blood pressure, respiratory rate, GCS, or Revised Trauma Score (RTS). 
Overall ED mortality in trauma patients decreased from 8.77 to $6.26 \%$ in the full cohort ( $p$ $=0.09$, OR 0.69 (95\% CI 0.46-1.05)). Patients with an initial GCS of 3-8, representing the severe injury subset, had the highest baseline mortality and showed a $60 \%$ reduction in the odds of mortality following the educational intervention. This mortality drop from 58.51 to $37.10 \%$ in the GCS $3-8$ subset was statistically significant $(p=0.009$, OR $0.42(95 \%$ CI $0.22-0.81)$ ). To put these results into perspective, these results were compared to available statistics in the hospital statistics department. ED mortality for all presenting patients (medical and surgical) was $1.14 \%$ in Phase 1 and $1.60 \%$ in Phase 2. In-hospital mortality (all medical and surgical patients) was available by the year only; hospital mortality was 7.8 $\%$ in 2010, $8.1 \%$ in 2011, $6.7 \%$ in 2012, and $6.6 \%$ in 2013.

Severe head injuries were further stratified into GCS 3-5 and GCS 6-8 based upon strata established in trauma $S B P$ systolic blood pressure, $D B P$ diastolic blood pressure, $R R$ respiratory rate, GCS Glasgow Coma Score, $R T S$ revised trauma score outcome studies in resource-limited settings [21]. Moderate head injuries were identified by GCS 9-12 and mild by GCS 13-15. The mortality decrease in the most severe (GCS 3-5) group from 84.09 to $62.96 \%$ was statistically significant $p=0.04$, OR 0.32 (95\% CI 0.10-0.99). The GCS 68 severe head injury subgroup showed a decrease from $36.00 \%$ before to $17.14 \%$ after, although this failed to show statistical significance $(p=0.06$, OR 0.37 (95\% CI 0.13-1.05).

Figure 2 shows the decrease in ED mortality seen in the full cohort and in the GCS 3-8 subset as well as stratified into the four GCS categories. Overall 30-day in-hospital mortality (including ED mortality) fell from 12.03 to $10.26 \%$ in the full cohort $(p=0.3)$ and from 65.96 to $50 \%$ in the GCS 3-8 subset $(p=0.05)$.

We found no statistically significant differences in the rates of early intubation, cervical collar use, or imaging studies in the overall cohort or in the GCS 3-8 subset, but there were differences when stratified by GCS. Tables 2 and 3 detail procedures performed in the ED and resource utilization in the full cohort (Table 2) and stratified by GCS (Table 3). In the GCS 3-5 group, the utilization of chest radiographs increased significantly from 11.36 to $33.33 \%(p=0.02)$, and the utilization of head CT scans increased from 38.64 to $66.67 \%$ ( $p$ $=0.02)$. Interestingly, utilization of head CT scans decreased in the GCS 9-12 strata (67.92 to $51.79 \%, p=0.04$ ), while spine imaging increased (6.60 to $17.86 \%, p=0.03$ ).

Evaluation of the full year following the intervention (November 2011-November 2012, $n=$ $1312)$ in comparison to the 6 months prior $(n=798)$ to the training showed a sustainable drop in ED mortality. The drop was not statistically significant in the overall cohort $(8.8 \%$ $(n=70)$ to $6.9 \%(n=90), p=0.11)$, whereas it was statistically significant in the GCS 3-8 subset $(58.5 \%(n=55)$ to $37.2 \%(n=48), p=0.0016)$. Overall 30-day hospital mortality showed similar drops (full cohort $12 \%(n=96)$ to $9.6 \%(n=126), p=0.08$, and GCS 3-8 subset $66 \%(n=62)$ to $49.6 \%(n=64), p=0.015)$. Evaluation of resource utilization in the full year following the educational intervention revealed a significant decrease in abdominal ultrasound utilization overall as well as a significant increase in head CT scans in the GCS 3-8 subset, from 58.5 to $73.6 \%(p=0.02)$. 


\section{Discussion}

Several authors suggest that training and retaining a healthcare workforce in LMIC is the most critical step toward achieving sustainable changes in socioeconomic development and that health outcomes are positively correlated with healthcare worker density [22, 23]. Trauma educational initiatives in LMIC have been shown to positively impact provider practices and knowledge [12,17-20], but their effect on patient outcomes has yet to described $[24,25]$.

Using single hospital data from the Rwanda injury registry to evaluate the impact of two trauma education courses, our study found that the mortality of injured patients with an initial GCS 3-8 significantly decreased over the 6 months following initiation of focused trauma education courses in the capital city of a low-income country. We have further demonstrated that the changes in mortality were sustained up to a year following the training initiation. Although a causal association between increased education efforts and decreased mortality makes teleological sense, such a change is likely multi-factorial.

While we hypothesized that any improvements in outcomes would be associated with improved resource utilization, the overall explanation for our observations may be complex as minimal differences in resource utilization were noted during the study period (some changes were noted in abdominal ultrasound and head CT over the one-year period). The two courses, which at the time were given only once, are ultimately a relatively small intervention in the setting of a huge multi-factorial challenge of injury in LMIC. There are many confounding factors that can explain resource utilization. One consideration may be a patient's ability to pay for treatment or studies. While over $90 \%$ of the Rwandan population has basic health insurance, patients and families are still required to pay a percentage of the cost of CT scan or X-ray prior to the study. Radiology equipment is often out of service for maintenance. Our study cannot specifically evaluate how a patient's ability to pay or equipment maintenance affected the resources utilized during the study period.

The ability to assume causation or even lack of impact in the cohorts examined should not be used as a reflection of course effectiveness within this complex environment. Clearly, such interventions are essential, but significant change will take a long time and will likely also be associated with other system-based changes and culture change in the care environment. The ATLS course and the TTT course in 2011 have been followed by other courses, on-the-ground educational partnerships, and a changing culture for emergency care. The TTT course, for example, has since been adopted by the Faculty of Medicine at the National University of Rwanda and administered over six times by Rwandan faculty since the initial course. Longterm multidisciplinary efforts that pair training with changes in resources and mentorship may be needed to produce broad and lasting changes. These focused education courses are very difficult to evaluate in isolation and should be seen as an important and necessary component of overall health system strengthening.

Injuries contribute to more global deaths each year than HIV/AIDS, malaria, and tuberculosis combined, accounting for $16 \%$ of the global burden of disease [12]. Poor access to healthcare services in LMIC means that a significant number of injured patients 
may never receive formal medical care [24]. Due to economic and resourcing constraints, formal pre-hospital emergency medical services may be limited or non-existent in lowincome countries, and so, interventions occur at the hospital level for those patients who do receive any care $[25,26]$. Therefore, hospitals and hospital staff are important targets for educational interventions. Expenses associated with implementation of formal courses like ATLS may limit their applicability in many low-resource settings, but the principles should be transferrable. This specific ATLS course was a demonstration course, and so was provided by volunteers at minimal cost to the Rwandan surgeons and nurses. At full cost, formal ATLS programs may be cost-prohibitive in resource-limited settings. Teaching hospitals in Nigeria and Ghana have developed their own trauma education courses derived from ATLS principles and adapted to local availability of materials and infrastructure as well as local injury epidemiology [27, 28]. Given that care is often provided by nonphysicians, the Canadian Network for International Surgery's (CNIS) Trauma Team Training (TTT) course exemplifies the move to develop a team-based approach rather than focusing solely on physicians [6].

Our study has many challenges such as data limitations within the registry itself, staff turnover, and resource limitations for healthcare providers. Reproducibility or sustained change is difficult to determine without further analysis and support of the registry. Furthermore, while all of the faculty surgeons trained have a role in resident and medical student education, they are not the primary care-givers when a patient first arrives in emergency. Any attributable effect from their training on ED outcomes is likely due to the fact that these providers are responsible for training and oversight of medical students, residents, and nurses who are the first medical providers in the ED. Therefore, integration of sustainable education into the healthcare system through team-based approaches and utilizing a train the trainer model, as has been done with the subsequent trauma courses in Rwanda, could be next steps. Education is seen by Ministries of Health, educators, and development organizations as the key component to sustainable change, but these interventions are VERY difficult to evaluate, particularly in low-resource settings with limited resources. Prior reports have focused on knowledge retention and subjective impact of these courses on the participants, but this evaluation specifically examined patient outcomes. Each course did collect participant feedback, but 6-month knowledge and retention scores are not available. While it is important that we evaluate programs systematically in order to ensure responsible stewardship of resources and time, these should be seen as an integral part of health system strengthening with a focus on long-term sustainability.

\section{Conclusions}

The mortality of severely injured patients decreased after initiation of focused trauma education courses in the capital of Rwanda. The explanation for this observation may be complex and multi-factorial as no significant increase in resource utilization was noted during the study period, nor was any decrease in overall mortality noted for the population as a whole. Long-term multidisciplinary efforts that pair training with changes in resources and mentorship may be needed to produce broad and lasting changes in the overall care system. 


\section{Acknowledgments}

Support for the Rwanda Injury Registry (local staff stipends, training, and registry printing) comes from a Fogarty International Clinical Research Fellowship, NIH and International Clinical Research Fellows Program at Vanderbilt University, USA (R24 TW007988) from February 2011-August 2012. Maintenance of the Rwanda Injury Registry is currently funded by the University of Virginia Department of Surgery. Salary for RTP (author) was funded by NIH 5-T32-AI-078875-03, PI: Robert G. Sawyer from July 1, 2012, to June 30, 2013.

\section{References}

1. World Health Organization (WHO). World report on road traffic injury prevention. World Health Organization; Geneva: 2004.

2. Lagarde E. Road traffic injury is an escalating burden in Africa and deserves proportionate research efforts. PLoS Med. 2007; 4:e170. [PubMed: 17593893]

3. Mathers CD, Loncar D. Projections of global mortality and burden of disease from 2002 to 2030. Plos Med. 2006; 3(11):e442. [PubMed: 17132052]

4. Ameratunga S, Hijar M, Norton R. Road-traffic injuries: confronting disparities to address a globalhealth problem. Lancet. 2006; 367(9521):1533-1540. [PubMed: 16679167]

5. Mock C, Joshipura M, Goosen J, Lormand JD, Maier R. Strengthening trauma systems globally: the essential trauma care project. J Trauma. 2005; 59(5):1243-1246. [PubMed: 16385310]

6. Bergman S, Deckelbaum D, Lett R, et al. Assessing the impact of the trauma team training program in Tanzania. J Trauma. 2008; 65(4):879-883. [PubMed: 18849806]

7. Jayaraman S, Sethi D. Advanced trauma life support training for ambulance crews. Cochrane Database Syst Rev. 2010; 8(1):CD003109.

8. Jayaraman S, Sethi D. Advanced trauma life support training for hospital staff. Cochrane Database Syst Rev. 2009; 2:CD004173. doi: 10.1002/14651858.CD004173.pub4. [PubMed: 19370594]

9. Husum H, Gilbert M, Wisborg T. Training pre-hospital trauma care in low-income countries: the 'Village University' experience. Med Teach. 2003; 25(2):142-148. [PubMed: 12745521]

10. Radvinsky DS, Yoon RS, Schmitt PJ, Prestigiacomo CJ, Swan KG, Liporace FA. Evolution and development of the advanced trauma life support (ATLS) protocol: a historical perspective. Orthopedics. 2012; 35(4):305-311. [PubMed: 22495839]

11. Vestrup JA, Stormorken A, Wood V. Impact of advanced trauma life support training on early trauma management. Am J Surg. 1988; 155(5):704-707. [PubMed: 3369629]

12. Ali J, Adam R, Butler AK, et al. Trauma outcome improves following the advanced trauma life support program in a developing country. J Trauma. 1993; 34(6):890-898. discussion 898-899. [PubMed: 8315686]

13. Kobusingye OC, Lett RR. Hospital-based trauma registries in Uganda. J Trauma. 2000; 48(3):498502. [PubMed: 10744292]

14. Mukhopadhyay B, Boniface R, Razek T. TRAUMA IN TANZANIA: Researching injury in a lowresource setting. Mcgill J Med. 2009; 12(2):27. [PubMed: 21152331]

15. Demyttenaere SV, Nansamba C, Nganwa A, Mutto M, Lett R, Razek T. Injury in Kampala, Uganda: 6 years later. Can J Surg. 2009; 52(5):E146-E150. [PubMed: 19865544]

16. Taye M, Munie T. Trauma registry in Tikur Anbessa Hospital, Addis Ababa, Ethiopia. Ethiop Med J. 2003; 41(3):221-226. [PubMed: 15227887]

17. Schultz CR, Ford HR, Cassidy LD, et al. Development of a hospital-based trauma registry in Haiti: an approach for improving injury surveillance in developing and resource-poor settings. J Trauma. 2007; 63(5):1143-1154. [PubMed: 17993964]

18. Rwanda National Institute of Statistics. National Population Projection, 2007-2022. National Census Commission; Kigali: 2009.

19. World Bank Country Data. World Bank; Washington, DC: 2013. Accessed online 4 February 2013

20. Chalya PL, Mabula JB, Dass RM, et al. Injury characteristics and outcome of road traffic crash victims at Bugando Medical Centre in Northwestern Tanzania. J Trauma Manag Outcomes. 2012; 6(1):1. [PubMed: 22321248] 
21. Benatar SR, Fleisher TE, Peter JC, et al. Treatment of head injuries in the public sector in South Africa. S Afr Med J. 2000; 90(8):790-793. [PubMed: 11022628]

22. Anyangwe SC, Mtonga C. Inequities in the global health workforce: the greatest impediment to health in sub-Saharan Africa. Int J Environ Res Public Health. 2007; 4(2):93-100. [PubMed: 17617671]

23. Chen L, Evans T, Anand S, et al. Human resources for health: overcoming the crisis. Lancet. 2004; 364(9449):1984-1990. [PubMed: 15567015]

24. Mock CN, nii-Amon-Kotei D, Maier RV. Low utilization of formal medical services by injured persons in a developing nation: health service data underestimate the importance of trauma. $\mathrm{J}$ Trauma. 1997; 42(3):504-511. discussion 511-503. [PubMed: 9095119]

25. Mock C, Kobusingye O, Joshipura M, Nguyen S, Arreola-Risa C. Strengthening trauma and critical care globally. Curr Opin Crit Care. 2005; 11(6):568-575. [PubMed: 16292061]

26. Mann NC, Mullins RJ, MacKenzie EJ, Jurkovich GJ, Mock CN. Systematic review of published evidence regarding trauma system effectiveness. J Trauma. 1999; 47(3 Suppl):S25-S33. [PubMed: 10496607]

27. Tortella BJ, Swan KG, Donahoo JS, et al. Trauma life support education: a didactic and caprine laboratory course for Nigerian physicians. Injury. 1996; 27(5):329-331. [PubMed: 8763286]

28. Mock CN, Quansah R, Addae-Mensah L, Donkor P. The development of continuing education for trauma care in an African nation. Injury. 2005; 36(6):725-732. [PubMed: 15910824] 


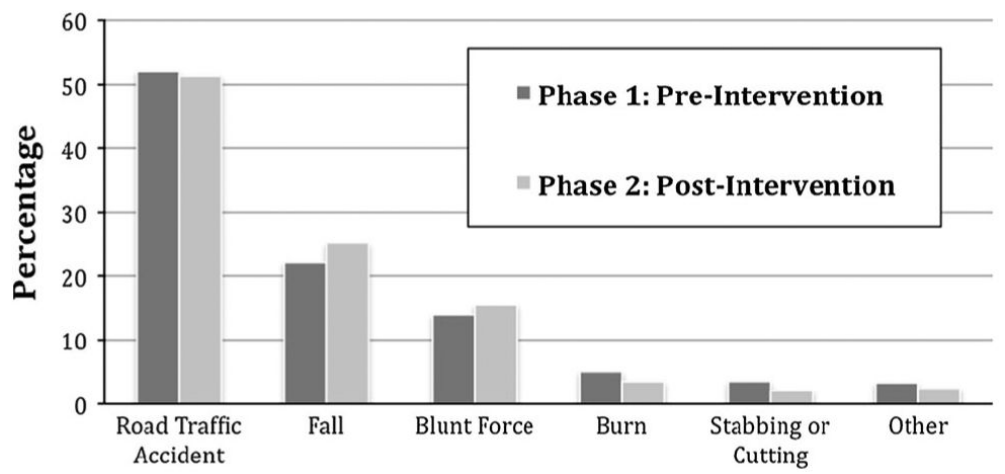

Fig. 1.

Cause of injury by phase. Road traffic crashes caused over $50 \%$ of the injuries in both phases. Falls and blunt force injuries were the next most common, with less than $10 \%$ due to other causes 


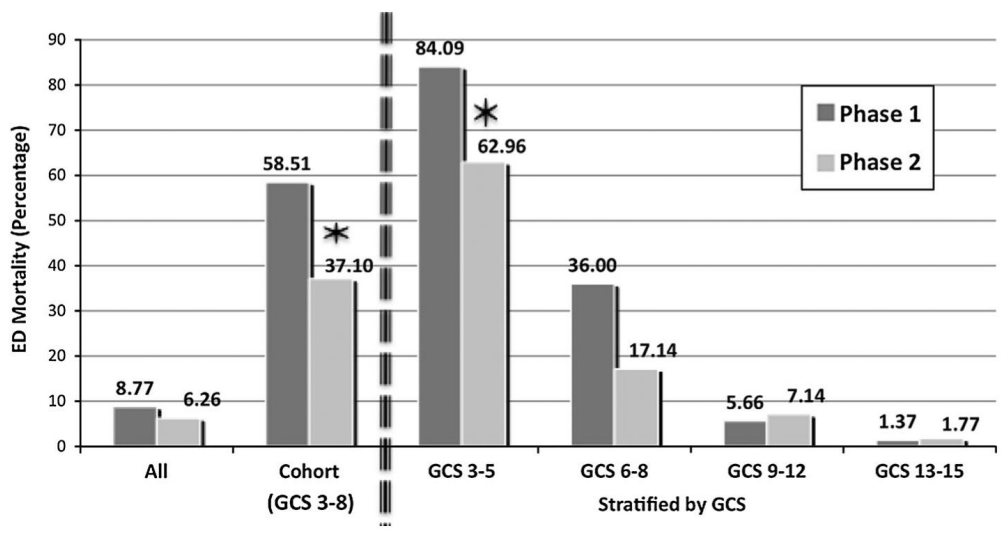

Fig. 2.

Mortality in the emergency department by phase. Overall ED mortality decreased from 8.77 to $6.26 \%$ after the educational intervention $[p=0.09$, OR 0.69 (95\% CI 0.46-1.05)]. For patients with an initial GCS of 3-8, mortality fell from 58.51 to $37.10 \%$ ( $p=0.009$, OR 0.42 (95 \% CI 0.22-0.81)). Stratifying all patients by GCS, the mortality decrease in the most severe (GCS 3-5) group from 84.09 to $62.96 \%$ was statistically significant $p=0.04$, OR 0.32 (95\% CI 0.10-0.99). The GCS 6-8 severe head injury subgroup showed a decrease from $36.00 \%$ before to $17.14 \%$ after, although this failed to show statistical significance $(p$ $=0.06$, OR $0.37(95 \%$ CI 0.13-1.05) 


\section{Table 1}

Comparing group characteristics—phase 1 versus phase 2

\begin{tabular}{|c|c|c|c|}
\hline Variable & Phase 1 & Phase 2 & $p$ value \\
\hline \multicolumn{4}{|l|}{ Age } \\
\hline Mean (SD) & $29.36(18.89)$ & 30.47 (20.14) & 0.65 \\
\hline Median (IQR) & $27(17-38)$ & $26(17-42)$ & \\
\hline \multicolumn{4}{|l|}{ Pulse } \\
\hline Mean (SD) & $87.83(20.67)$ & 89.58 (23.98) & 0.34 \\
\hline Median (IQR) & $84(75-100)$ & $85(75-101)$ & \\
\hline \multicolumn{4}{|l|}{ SBP } \\
\hline Mean (SD) & 116.18 (18.89) & 118.09 (17.34) & 0.13 \\
\hline Median (IQR) & $116(105-125.5)$ & $120(110-128)$ & \\
\hline \multicolumn{4}{|l|}{ DBP } \\
\hline Mean (SD) & $69.83(14.14)$ & 68.75 (13.94) & 0.34 \\
\hline \multicolumn{4}{|l|}{$\mathrm{RR}$} \\
\hline Mean (SD) & $21.09(3.75)$ & $21.23(3.31)$ & 0.29 \\
\hline \multicolumn{4}{|l|}{ GCS } \\
\hline Mean (SD) & $13.16(3.35)$ & $13.43(3.07)$ & 0.39 \\
\hline \multicolumn{4}{|l|}{ RTS } \\
\hline Mean (SD) & $7.38(0.86)$ & $7.26(0.96)$ & 0.13 \\
\hline \multicolumn{4}{|l|}{ Sex } \\
\hline$\%$ Male $(n)$ & $77.69(620)$ & $75.3(433)$ & 0.30 \\
\hline \multicolumn{4}{|l|}{ Penetrating } \\
\hline$\%(n)$ & $4.01(32)$ & $2.61(15)$ & 0.16 \\
\hline \multicolumn{4}{|l|}{ GCS 3-8 } \\
\hline$\%(n)$ & $11.78(94)$ & $10.78(62)$ & 0.57 \\
\hline
\end{tabular}


Table 2

Interventions and resource utilization—phase $1(n=798)$ versus phase $2(n=575)$

\begin{tabular}{lllll}
\hline & Phase 1 \% $(\boldsymbol{n})$ & Phase 2 \% $(\boldsymbol{n})$ & $\boldsymbol{p}$ value & OR $(\mathbf{9 5} \% \mathbf{C I})$ \\
\hline Intubation & $6.77(54)$ & $6.96(40)$ & 0.89 & $1.03(0.67-1.57)$ \\
Cervical collar & $5.26(42)$ & $6.96(40)$ & 0.19 & $1.35(0.86-2.10)$ \\
Chest X-ray & $16.54(132)$ & $18.78(108)$ & 0.28 & $1.17(0.88-1.54)$ \\
Pelvis X-ray & $10.4(83)$ & $13.57(78)$ & 0.07 & $1.35(0.97-1.88)$ \\
CT head & $22.18(177)$ & $22.26(128)$ & 0.97 & $1.00(0.78-1.30)$ \\
Abdominal US & $6.64(53)$ & $4.35(25)$ & 0.07 & $0.64(0.39-1.04)$ \\
Extremity X-ray & $43.48(347)$ & $45.74(263)$ & 0.41 & $1.10(0.88-1.36)$ \\
Spine imaging & $11.03(88)$ & $13.74(79)$ & 0.13 & $1.29(0.93-1.78)$ \\
Body CT & $0.50(4)$ & $1.04(6)$ & 0.34 & $2.09(0.59-7.45)$ \\
\hline
\end{tabular}

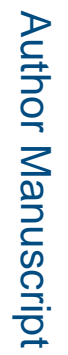

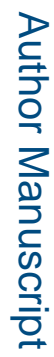

World J Surg. Author manuscript; available in PMC 2016 April 01. 
Table 3

Interventions and resource utilization by GCS Strata

\begin{tabular}{|c|c|c|c|c|}
\hline & Phase $1 \%(n)$ & Phase $2 \%(n)$ & $p$ value & OR $(95 \% \mathrm{CI})$ \\
\hline \multicolumn{5}{|c|}{ (a) Severe head injury patients (GCS 3-5)-phase $1(n=44)$ versus phase $2(n=27)$} \\
\hline Intubation & $45.45(20)$ & $51.85(14)$ & 0.60 & $1.29(0.49-3.38)$ \\
\hline Cervical collar & $6.82(3)$ & $14.81(4)$ & 0.27 & $2.38(0.49-11.56)$ \\
\hline Chest X-ray & $11.36(5)$ & $33.33(9)$ & 0.02 & $3.90(1.14-13.31)$ \\
\hline Pelvis X-ray & $9.09(4)$ & $14.81(4)$ & 0.46 & $1.74(0.40-7.62)$ \\
\hline CT head & $38.64(17)$ & $66.67(18)$ & 0.02 & $3.18(1.16-8.67)$ \\
\hline Abdominal US & $4.55(2)$ & $0(0)$ & 0.26 & \\
\hline Extremity X-ray & $4.55(2)$ & $7.41(2)$ & 0.61 & $1.68(0.22-12.68)$ \\
\hline Spine imaging & $9.09(4)$ & $18.52(5)$ & 0.25 & $2.27(0.55-9.35)$ \\
\hline Body CT & $0(0)$ & $3.70(1)$ & & \\
\hline \multicolumn{5}{|c|}{ (b) Severe head injury patients (GCS 6-8)-phase $1(n=50)$ versus phase $2(n=35)$} \\
\hline Intubation & $50.00(25)$ & $42.86(15)$ & 0.52 & $0.75(0.31-1.79)$ \\
\hline Cervical collar & $18.00(9)$ & $22.86(8)$ & 0.58 & $1.35(0.46-3.93)$ \\
\hline Chest X-ray & $20.00(10)$ & $20.00(7)$ & 1.00 & $1.00(0.34-2.94)$ \\
\hline Pelvis X-ray & $8.00(4)$ & $8.57(3)$ & 0.92 & $1.08(0.23-5.15)$ \\
\hline CT head & $76.00(38)$ & $68.57(24)$ & 0.45 & $0.69(0.26-1.81)$ \\
\hline Abdominal US & $8.00(4)$ & $0(0)$ & 0.09 & \\
\hline Extremity X-ray & $8.00(4)$ & 8.57 (3) & 0.92 & $1.08(0.23-5.15)$ \\
\hline Spine imaging & $16.00(8)$ & $20.00(7)$ & 0.63 & $1.31(0.43-4.02)$ \\
\hline Body CT & $0(0)$ & $0(0)$ & & \\
\hline \multicolumn{5}{|c|}{ (c) Moderate head injury patients (GCS 9-12)—phase $1(n=106)$ versus phase $2(n=56)$} \\
\hline Intubation & $3.77(4)$ & $7.14(4)$ & 0.35 & $1.96(0.47-8.16)$ \\
\hline Cervical collar & $6.60(7)$ & $10.71(6)$ & 0.36 & $1.70(0.54-5.32)$ \\
\hline Chest X-ray & $19.81(21)$ & $23.21(13)$ & 0.61 & $1.22(0.56-2.68)$ \\
\hline Pelvis X-ray & $9.43(10)$ & $8.93(5)$ & 0.92 & $0.94(0.31-2.90)$ \\
\hline CT head & $67.92(72)$ & $51.79(29)$ & 0.04 & $0.51(0.26-0.99)$ \\
\hline Abdominal US & $1.89(2)$ & $1.79(1)$ & 0.96 & $0.95(0.08-10.66)$ \\
\hline Extremity X-ray & $16.98(18)$ & $17.86(10)$ & 0.89 & $1.06(0.45-2.49)$ \\
\hline Spine imaging & $6.60(7)$ & $17.86(10)$ & 0.03 & $3.07(1.10-8.59)$ \\
\hline Body CT & $1.89(2)$ & $0(0)$ & & \\
\hline \multicolumn{5}{|c|}{ (d) Mild head injury patients (GCS 13-15)-phase $1(n=1034)$ versus phase $2(n=583)$} \\
\hline Intubation & $0.51(3)$ & $1.11(5)$ & 0.28 & $2.17(0.52-9.12)$ \\
\hline Cervical collar & $3.77(22)$ & $4.88(22)$ & 0.38 & $1.31(0.71-2.39)$ \\
\hline Chest X-ray & $15.61(91)$ & $17.29(78)$ & 0.47 & $1.13(0.81-1.57)$ \\
\hline Pelvis X-ray & $10.98(64)$ & $14.19(64)$ & 0.12 & $1.34(0.93-1.94)$ \\
\hline CT Head & $8.23(48)$ & $67.74(42)$ & 0.24 & $1.49(0.76-2.92)$ \\
\hline Abdominal US & $7.38(43)$ & $12.42(56)$ & 0.03 & $1.58(1.05-2.37)$ \\
\hline Extremity X-ray & $54.55(318)$ & $54.32(245)$ & 0.94 & $0.99(0.77-1.27)$ \\
\hline Spine imaging & $11.49(67)$ & $12.64(57)$ & 0.57 & $1.11(0.76-1.62)$ \\
\hline
\end{tabular}




\begin{tabular}{lllll}
\hline & Phase 1\% $(\boldsymbol{n})$ & Phase 2 \% $(\boldsymbol{n})$ & $\boldsymbol{p}$ value & OR $(\mathbf{9 5} \% \mathbf{C I})$ \\
\hline Body CT & $0.34(2)$ & $1.11(5)$ & 0.14 & $3.26(0.63-16.86)$ \\
\hline
\end{tabular}

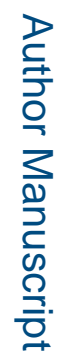

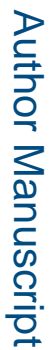

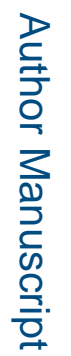

World J Surg. Author manuscript; available in PMC 2016 April 01. 